\title{
Erlotinib treatment in pretreated patients with non-small cell lung cancer: A Phase II study
}

\author{
G.P. STATHOPOULOS ${ }^{1}$, D. TRAFALIS ${ }^{1,4}$, J. DIMITROULIS ${ }^{2}$, A. ATHANASIOU ${ }^{3}$, \\ J. KOUTANTOS ${ }^{1}$ and A. ANAGNOSTOPOULOS ${ }^{1}$
}

\author{
${ }^{1}$ First Oncology Clinic, Errikos Dunant Hospital; ${ }^{2}$ Sixth Clinic, Hospital of Thoracic Diseases, Athens; ${ }^{3}$ Oncology Clinic, \\ Metaxa Hospital, Piraeus; ${ }^{4}$ Department of Pharmacology, Medical School, University of Athens, Athens, Greece
}

Received October 5, 2009; Accepted December 23, 2009

DOI: 10.3892/ol_00000059

\begin{abstract}
Erlotinib is an oral, small-molecule targeting therapy that inhibits epidermal growth factor tyrosine kinase receptors. Erlotinib has been administered for the treatment of advanced pancreatic cancer and non-small cell lung cancer. In the present trial, erlotinib was administered as second-line monotherapy in pretreated patients with advanced non-small cell lung cancer. Our objectives were to determine response, survival and toxicity. Fifty-four patients pretreated with cisplatin or its analogue-based combinations were evaluated. The disease stage of the patients was IIIB and IV. Thirty-eight patients were male, 16 were female, the median age was 65 years, and the WHO performance status was 0-2. Twenty-five cases were adenocarcinomas, 19 squamous cell carcinomas and 10 were undifferentiated. Erlotinib was administered at a dose of $150 \mathrm{mg}$ daily. In case of intolerable adverse reactions, the dose was either reduced to $100 \mathrm{mg}$ daily or treatment was interrupted for a maximum of two weeks. A partial response was observed in $10(18.52 \%)$ and stable disease in 40 (74.07\%) patients. The median time to disease progression was 3 months (95\% CI 1.7-10.3), and the median survival was 6 months. Concerning toxicity, 53 patients $(98.15 \%)$ developed a grade 1-2 skin rash, and $1(1.85 \%)$ grade 3 . Diarrhea occurred in $9(16.67 \%)$ patients, nausea and vomiting in $4(7.41 \%)$ and gastritis in $2(3.70 \%)$. The majority of patients tolerated the erlotinib treatment. Of note were the $18.52 \%$ response rate and $74.07 \%$ stable disease.
\end{abstract}

\section{Introduction}

Erlotinib is an oral, small-molecule targeting therapy that inhibits the epidermal growth factor receptor (EGFR) of tyrosine kinase, blocking signal transduction pathways implicated in the proliferation and survival of cancer cells

Correspondence to: Dr G.P. Stathopoulos, Semitelou 2A, 11528 Athens, Greece

E-mail: dr-gps@ath.forthnet.gr

Key words: erlotinib, non-small cell lung cancer
(1). EGFR is associated with cellular processes leading to tumorigenesis $(2,3)$. Data exist concerning erlotinib administration for malignant tumors, mainly pancreatic cancer, in combination with another cytotoxic agent, as well as for non-small cell lung cancer (NSCLC) in a large number of patients as a second-line treatment (4). Erlotinib has provided a survival benefit for advanced NSCLC patients $(5,6)$. The data reported by two Phase III studies led to US Food and Drug Administration (FDA) approval for the use of erlotinib in NSCLC patients after first-line chemotherapy failure. A survival benefit was demonstrated in patients with advanced pancreatic cancer when erlotinib was combined with gemcitabine vs. gemcitabine alone (7). A survival benefit was even shown in several subsets of NSCLC patients such as those with squamous cell carcinoma, smokers and males, where gefitinib did not appear to be active (5).

Serious adverse reactions are uncommon. The most common side effects are skin rash and serious grade 3-4 anorexia followed by fatigue, vomiting and stomatitis which were reported to be less than 1\%. Grade 3-4 diarrhea was also less than $1 \%(6)$.

The present study involves erlotinib monotherapy in pretreated patients with advanced NSCLC. The primary objective was to determine the response rate and survival in pretreated patients, and the secondary objective was to determine toxicity.

\section{Materials and methods}

Patient eligibility. Eligibility for the study involved histologically or cytologically confirmed NSCLC, disease staging and a defined inoperable stage IIIB or IV. Stage IIIA was only included in case of chronic respiratory insufficiency which did not permit surgery. A requirement was that patients had to have undergone one or two lines of prior chemotherapy. Radiation therapy was not excluded as a previous treatment. Bidimensionally measurable disease criteria were: physical examination, X-rays, computed tomography (CT), World Health Organization (WHO) performance status 0-2, expected survival $\geq 12$ weeks, adequate bone marrow reserves (leukocyte count $\geq 3,500 \mu \mathrm{l}^{-1}$, platelet count $\geq 100,000 \mu \mathrm{l}^{-1}$ and hemoglobin $\geq 10 \mathrm{~g} \mathrm{dl}^{-1}$ ), adequate renal function (serum creatinine $\leq 1.5 \mathrm{mg} / \mathrm{dl}^{-1}$ and serum transaminases $\leq 3$ times the upper 
normal limit or $\leq 5$ times the upper normal limit in cases of liver metastases) and age $\geq 18$ years. In cases of central nervous system involvement or any secondary malignancy, patients were excluded. The study was conducted with the approval of our institutional review boards, and all patients gave their written informed consent before enrollment.

Treatment. Erlotinib was administered at a dose of $150 \mathrm{mg}$ ( 1 tablet) per day. In case of adverse reactions, treatment was either reduced to $100 \mathrm{mg}$ or interrupted for a maximum of two weeks. Otherwise, treatment was continued until disease progression, intolerable toxicity or refusal to continue.

Previous treatment. Before entering the study, patients had received chemotherapy based on cisplatin (44 patients) or carboplatin (5 patients). The second agent of the combination was paclitaxel (40 patients), vinorelbine (4 patients), gemcitabine (3 patients) or etoposide ( 2 patients). Eleven patients underwent second-line chemotherapy 3-9 months after the end of the first-line treatment. The agents administered for the second-line chemotherapy included docetaxel, pemetrexed or etoposide. Five patients received a combination of the first two aforementioned agents and 6 received a single treatment of one of the three agents (Table I).

Baseline and treatment assessment and evaluation. Before enrollment, the patients underwent physical examination, tumor measurement and evaluation, WHO performance status, electrocardiogram, full blood count, renal and liver function tests and urinalysis. Staging was determined by chest and abdominal CT scans, bone scan and occasional magnetic resonance imaging. Blood counts, blood urea and serum creatinine were measured before each treatment administration and every 3 weeks thereafter. During the treatment period, radiologic tests were conducted: a chest X-ray once every 3 weeks and CT once every 2 months, or whenever there were signs of disease progression. Imaging-based evaluation was used to assess response. A complete response (CR) was defined as the disappearance of all measurable disease, confirmed at 4 weeks at the earliest. A partial response (PR) was defined as a $30 \%$ decrease, confirmed at 4 weeks at the earliest. In stable disease (SD), neither PR nor progressive disease (PD) criteria were met; PD involved a $20 \%$ increase in tumor burden but no $\mathrm{CR}, \mathrm{PR}$ or SD before increased disease. Response data were based on the response evaluation criteria in solid tumors (8). A two-step deterioration in performance status, a $>10 \%$ loss in pretreatment weight or increasing symptoms did not by themselves constitute progression of the disease. However, the appearance of these signs was followed by a new evaluation of the extent of the disease. Responses had to be maintained for at least 4 weeks and to be confirmed by two independent radiologists and two experienced oncologists.

Statistical design. This was an expected two-step Phase II study and an intent-to-treat analysis. According to the trial design, 30 patients were to be enrolled during the first part of the study and if an objective response rate of $<15 \%$ was achieved, the treatment would have been abandoned; otherwise, 20 additional patients were to be enrolled. The primary objective of the study was to determine the efficacy
Table I. Patient characteristics.

n $(\%)$

Patients enrolled

Patients assessable

Gender

Male

$38(70.37)$

Female

Age

Median

Range

WHO performance status

0

1

2

Disease stage

IIIB

IV

Histology

Adenocarcinoma

Squamous cell carcinoma

Undifferentiated

$10(18.52)$

Prior treatment

First-line

Cisplatin-paclitaxel

Cisplatin-vinorelbine

Carboplatin-gemzar

Carboplatin-etoposide

Second-line

Docetaxel-gemcitabine

Carboplatin-etoposide

Docetaxel

Vinorelbine

Pemetrexed

2 (18.18)

of the regimen with respect to response and survival, and the secondary objective was to determine the toxicity. Survival was calculated from the day of enrollment until death or the end of the study. The median probability of survival was estimated by the Kaplan-Meier method; confidence intervals (CIs) for response rates were calculated using methods for the exact binomial CI.

\section{Results}

From April 2007 to December 2008, 54 patients with advanced or metastatic NSCLC were enrolled in the present trial. The patients were considered evaluable for response, toxicity and survival. Patient characteristics at baseline are shown in Table I. The median age was 65 years (range 37-81) and their WHO performance status was 0-2. All patients had undergone prior chemotherapy mainly based on cisplatin or a carboplatin combination. Eleven of the 54 patients had under- 
Table II. Response rate.

\begin{tabular}{lr}
\hline & $\mathrm{n}(\%)$ \\
\hline Partial response & $10(18.52)$ \\
Stable disease & $40(74.07)$ \\
Disease progression & $4(7.41)$ \\
\hline
\end{tabular}

gone second-line chemotherapy before entering the present trial. The first- and second-line treatment is shown in Table I. The median duration of treatment was 4 months (range 1.5-18); 40 patients $(70.07 \%)$ underwent $4-18$ months of treatment.

Response. Of the 54 assessable patients, an objective response rate was observed in $10(18.52 \%)$ patients, while the median duration of response was 6 months (range 3-8). Stable disease was observed in 40 patients $(74.07 \%)$ and disease progression in 4 patients $(7.41 \%)$. The response data are documented in Table II.

Survival data. At the end of the study, 15 of the 54 patients were still alive $(27.77 \%)$. The median follow-up was 8 months (range 3-20). The median survival time was 6 months (95\% CI 1.7-10.3). Fig. 1 shows the Kaplan-Meier survival curve. The median time to tumor progression (TTP) was 3 months $(95 \%$ CI 1.9-4.1). TTP is shown in Fig. 2 (Kaplan-Meier).

Toxicity. Grade 1-2 skin rash was the main adverse reaction observed in 53 of the 54 patients $(98.15 \%)$. Grade 3 skin rash was observed in 2 patients $(3.70 \%)$ and this proved to be intolerable. Thus, the dose of erlotinib was decreased from 150 to $100 \mathrm{mg}$. Other non-hematologic toxicities were grade 1-2 diarrhea in 9 patients (16.66\%), nausea and vomiting in $4(7.41 \%)$ and gastritis in $2(3.70 \%)$. Hematologic toxicity (leukopenia and thrombocytopenia) was not observed in 50 of the 54 patients $(92.59 \%)$.

\section{Discussion}

One of the first growth factors discovered was the epidermal growth factor (EGF) (9). It is a protein which binds to a cell surface growth factor receptor, the EGFR. In binding to the receptor, EGF either induces cell proliferation or differentiation in mammalian cells (10).

The binding of a ligand to the EGFR induces conformational changes within the receptor that increases the catalytic activity of its intrinsic tyrosine kinase, resulting in autophosphorylation which is necessary for biological activity (11,12). Protein tyrosine kinase activity plays a key role in the regulation of cell proliferation and differentiation (13). A large number of deletions of the EGFR in RNA have been observed in a number of neoplasias such as glioblastoma in non-small cell lung carcinomas, breast cancer, pediatric gliomas, medulloblastomas and ovarian carcinomas (13). Overexpression of mRNA and/or the protein encoded by the EGFR gene has been observed in many types of human malignancies (14), including breast (15), gastric, colorectal (16) and bladder cancer (17). In NSCLC, EGFR expression

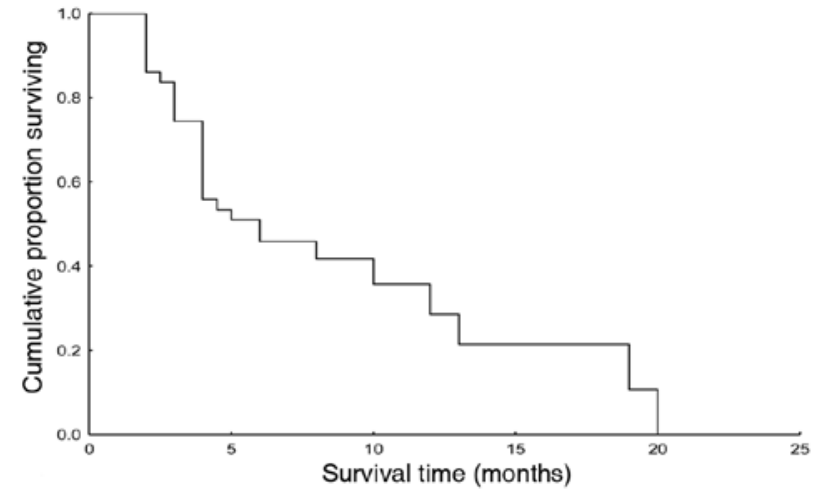

Figure 1. Kaplan-Meier survival curve.

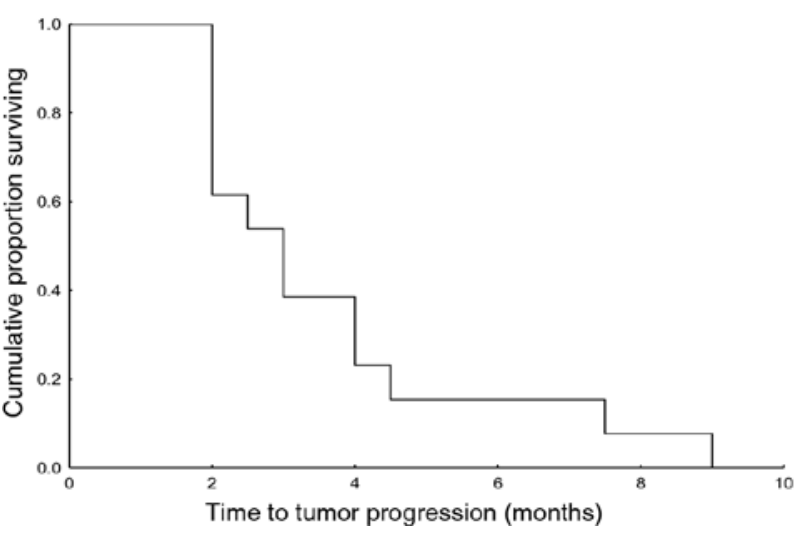

Figure 2. Kaplan-Meier curve of time to tumor progression.

at percentages varying from 30 to $70 \%$ has been reported $(18,19)$. Erlotinib is an anti-EGFR targeting agent; studies have already been performed and reports concerning its value have been documented $(20,21)$. However, despite the fact that targeting therapy has been administered in a considerable number of clinical trials over the recent years, there are many unanswered questions related to the failure to achieve the expected success and to explain certain adverse reactions or complications. Tumors are likely to express variable but excessive numbers of HER1/EGFRs. Unless all receptors are effectively inhibited from initiating signaling, there is likely to be sufficient residual tumorigenic activity to maintain disease (22). Evidence, although unconfirmed, suggests that cancer types become dependent on one or more specific elements of the cell signaling circuit, requiring their continued presence in order to remain malignant (23).

The results of the present trial showed that the response rate is higher than that of the 8.5 and $9.5 \%$ reported in previous studies $(5,6)$. Of note is the high disease stability (74.07\%) and the median TTP of 4 months. Three other studies reported a) a response rate of $13 \%$, a SD rate of $54 \%$ and a progression-free survival (PFS) of 9.7 weeks in 3,338 patients (24); b) PR 9\%, SD 67\% and PFS 12.3 weeks in 4,002 patients (25); and c) PR 12\%, SD 56\% and PFS 14.3 weeks in 6,809 patients (26).

Erlotinib may be an eligible second-line treatment for NSCLC patients. The majority of patients tolerate the treatment and adverse reactions. Low toxicity increases the 
tendency to support the use of erlotinib in pretreated NSCLC patients.

\section{References}

1. Baselga $\mathrm{J}$ and Arteaga CL: Critical update and emerging trends in epidermal growth factor receptor targeting in cancer. J Clin Oncol 23: 2445-2459, 2005.

2. Jänne PA, Engelman JA and Johnson BE: Epidermal growth factor receptor mutations in non-small cell lung cancer: implications for treatment and tumor biology. J Clin Oncol 23: 3227-3234, 2005.

3. Swinson DE, Cox G and O'Byrne KJ: Coexpression of epidermal growth factor receptor with related factors is associated with poor prognosis in non-small cell lung cancer. $\mathrm{Br} \mathrm{J}$ Cancer 91: 1301-1307, 2004.

4. Comis RL: The current situation: erlotinib (Tarceva) and gefitinib (Iressa) in non-small cell lung cancer. Oncologist 10: 467-470, 2005.

5. Byoung C-C, Im C-K, Park M-S, et al: Phase II study of erlotinib in advanced non-small cell lung cancer after failure of gefitinib. J Clin Oncol 25: 2528-2533, 2007.

6. Shepherd FA, Pereira JR, Ciuleanu T, et al: Erlotinib in previously treated non-small cell lung cancer. N Engl J Med 353: 123-132, 2005.

7. Moore MJ, Goldstein D, Hamm J, et al: Erlotinib plus gemcitabine compared with gemcitabine alone in patients with advanced pancreatic cancer: a Phase III trial of the National Cancer Institute of Canada Clinical Trials Group. J Clin Oncol 25: 1960-1966, 2007.

8. Therasse P, Arbuck SG, Eisenhawer EA, et al: New guidelines to evaluate the response to treatment in solid tumors. J Natl Cancer Inst 92: 205-216, 2000.

9. Cohen S: The epidermal growth factor (EGF). Cancer 51: 1787-1791, 1983.

10. Yarolen Y and Schlessinger J: Epidermal growth factor induces rapid reversible aggregation of the purified epidermal growth factor receptor. Biochemistry 26: 1443-1451, 1987.

11. Hsuan JJ: Oncogene regulation by growth factors. Anticancer Res 13: 2521-2522, 1993.

12. Soler C, Beguinot L and Carpenter G: Individual epidermal growth factor receptor autophosphorylation sites do not stringently define association motifs for several SH-2 containing proteins. J Biol Chem 269: 12320-12324, 1994.
13. Voldborg RB, Damstrup L, Spang-Thomsen M, et al: Epidermal growth factor receptor (EGFR) and EGFR mutations, function and possible role in clinical trials. Ann Oncol 8: 1197-1206, 1997.

14. Ann J-H, Kim S-W, Hong S-M, et al: Epidermal growth factor receptor (EGFR) expression in operable non-small cell lung carcinoma. J Korean Med Sci 19: 529-535, 2004.

15. Sainsbury JRC, Nicholson S, Angus B, et al: Epidermal growth factor receptor status of histological sub-types of breast cancer. Br J Cancer 58: 458-460, 1988.

16. Yasui W, Sumiyoshi H, Hata J, et al: Expression of epidermal growth factor receptor in human gastric and colonic carcinomas. Cancer Res 48: 137-141, 1988.

17. Smith K, Fenelly JA, Neal DE, et al: Characterization and quantitation of the epidermal growth factor receptor in invasive and superficial bladder tumors. Cancer Res 49: 5810-5815, 1989.

18. Veale D, Kerr N, Gibson GJ, et al: Characterization of epidermal growth factor receptor in primary human non-small cell lung cancer. Cancer Res 49: 1313-1317, 1989.

19. Fujino S, Enokibori T, Tezuka N, et al: A comparison of epidermal growth factor receptor levels and other prognostic parameters in non-small cell lung cancer. Eur J Cancer 32A: 2070-2074, 1996.

20. Hortobagyi GN and Santer G: Challenges and opportunities for erlotinib (Tarceva). What does the future hold? Semin Oncol 30 (Suppl 7): 47-53, 2003.

21. Arteaga CL: The epidermal growth factor receptor: from mutant oncogene in nonhuman cancers to therapeutic target in human neoplasias. J Clin Oncol 19: S32-S40, 2001.

22. Perez-Soler R: HER1/EGFR targeting: refining the strategy. Oncologist 9: 58-67, 2004.

23. Weinstein IB: Addiction to oncogenes - the Achilles heal of cancer. Science 297: 63-64, 2002.

24. Crino L, Eberhardt W, Boyer M, et al: Erlotinib as second-line therapy in patients (PTS) with advanced non-small cell lung cancer (NSCLC) and good performance status: interim analyses from the TRUST Study. Abstract Book 33rd ESMO Congress, Suppl 8: 264, 2008

25. Groen H, Barata F, McDermott R, et al: Efficacy of erlotinib in $>4000$ patients (PTS) with advanced non-small cell lung cancer (NSCLC): analysis of the European subpopulation on the TRUST Study. Abstract Book 33rd ESMO Congress, Suppl 8: 266, 2008.

26. Reck M, Mali P, Arrieta O, et al: Global efficacy and safety results from the TRUST study of erlotinib monotherapy in $>7000$ patients (PTS) with non-small cell lung cancer (NSCLC). Abstract Book 33rd ESMO Congress, Suppl 8: 262, 2008. 